\title{
Coherent representations of subjective spatial position in primary visual cortex and hippocampus
}

\author{
Aman B. Saleem ${ }^{1,2 \dagger}$, E. Mika Diamanti ${ }^{1,3 \dagger}$, Julien Fournier ${ }^{1}$, Kenneth D. Harris $^{4^{*}}$ \& Matteo Carandini ${ }^{1^{*}}$
}

\begin{abstract}
A major role of vision is to guide navigation, and navigation is strongly driven by vision ${ }^{1-4}$. Indeed, the brain's visual and navigational systems are known to interact ${ }^{5,6}$, and signals related to position in the environment have been suggested to appear as early as in visual corte ${ }^{6,7}$. To establish the nature of these signals we recorded in primary visual cortex (V1) and in the CA1 region of the hippocampus while mice traversed a corridor in virtual reality. The corridor contained identical visual landmarks in two positions, so that a purely visual neuron would respond similarly in those positions. Most V1 neurons, however, responded solely or more strongly to the landmarks in one position. This modulation of visual responses by spatial location was not explained by factors such as running speed. To assess whether the modulation is related to navigational signals and to the animal's subjective estimate of position, we trained the mice to lick for a water reward upon reaching a reward zone in the corridor. Neuronal populations in both CA1 and V1 encoded the animal's position along the corridor, and the errors in their representations were correlated. Moreover, both representations reflected the animal's subjective estimate of position, inferred from the animal's licks, better than its actual position. Indeed, when animals licked in a given location - whether correct or incorrect - neural populations in both V1 and CA1 placed the animal in the reward zone. We conclude that visual responses in V1 are tightly controlled by navigational signals, which are coherent with those encoded in hippocampus, and reflect the animal's subjective position in the environment. The presence of such navigational signals as early as in a primary sensory area suggests that these signals permeate sensory processing in the cortex.
\end{abstract}

To characterise the influence of spatial position on the visual responses of area V1 we took transgenic mice expressing the calcium indicator GCaMP6 in excitatory cells and placed them in a $100 \mathrm{~cm}$ corridor in virtual reality (VR; Figure 1a). The corridor had four prominent landmarks, spaced $20 \mathrm{~cm}$ apart: a grating and a plaid, and then again a grating and a plaid. The repetition of landmarks created two visuallymatching segments of the corridor, separated by 40 $\mathrm{cm}$ (Figure 1a, b; Supplementary Figure 1). We identified V1 based on the retinotopic map of the cortical surface, measured using wide-field imaging ${ }^{8}$ (Figure 1c). We then pointed a two-photon microscope on regions in medial $\mathrm{V} 1$, focusing our analysis on neurons with receptive field centres $>40^{\circ}$ azimuth (Figure 1c), which were driven as the mouse passed the landmarks. As expected, given the repetition of visual scenes in the two segments of the corridor, some V1 neurons had a response profile with two equal peaks spaced $40 \mathrm{~cm}$ apart (Figure 1d). Other V1 neurons, however, responded very differently to the same visual stimuli in the two segments (Figure $1 \mathrm{~d}$ ). These results indicate that visual activity in V1 can be strongly modulated by the animal's spatial position in an environment.

This modulation of visual responses by spatial position occurred in the majority of $\mathrm{V} 1$ neurons (Figure 1e-g). We imaged the activity of 8,610 V1 neurons across 18 sessions in 4 mice. We selected neurons $(n=4,958)$ with receptive field centres $>40^{\circ}$ azimuth and activity significantly modulated by position in the corridor, and divided the trials in half, using the odd trials to find the position where each neuron fired maximally. The resulting representation reveals a striking preference of V1 neurons for spatial position, with most neurons giving much stronger responses in one position than in the visually matching position $40 \mathrm{~cm}$ away (Figure 1e). To quantify this preference while avoiding any circularity, we used the data obtained in the other half of the trials (even trials). These data showed that the preference for spatial position was robust (Figure 1f). Among the neurons that responded when the animal traversed the two visually-matching segments $(n=2,422)$, the responses to the landmarks $40 \mathrm{~cm}$ from the preferred position were substantially smaller than the responses at the preferred position (Figure 1g; Supplementary Figure 2): the median ratio of their responses was $0.61 \pm 0.31$ ( \pm m.a.d.). Modulation of visual responses by spatial position therefore reflected a reliable and widespread preference of individual V1 cells for one of the two locations.

The modulation of $\mathrm{V} 1$ responses by spatial position could not be explained by classical visual responses, or by deviations in pupil position and diameter, 

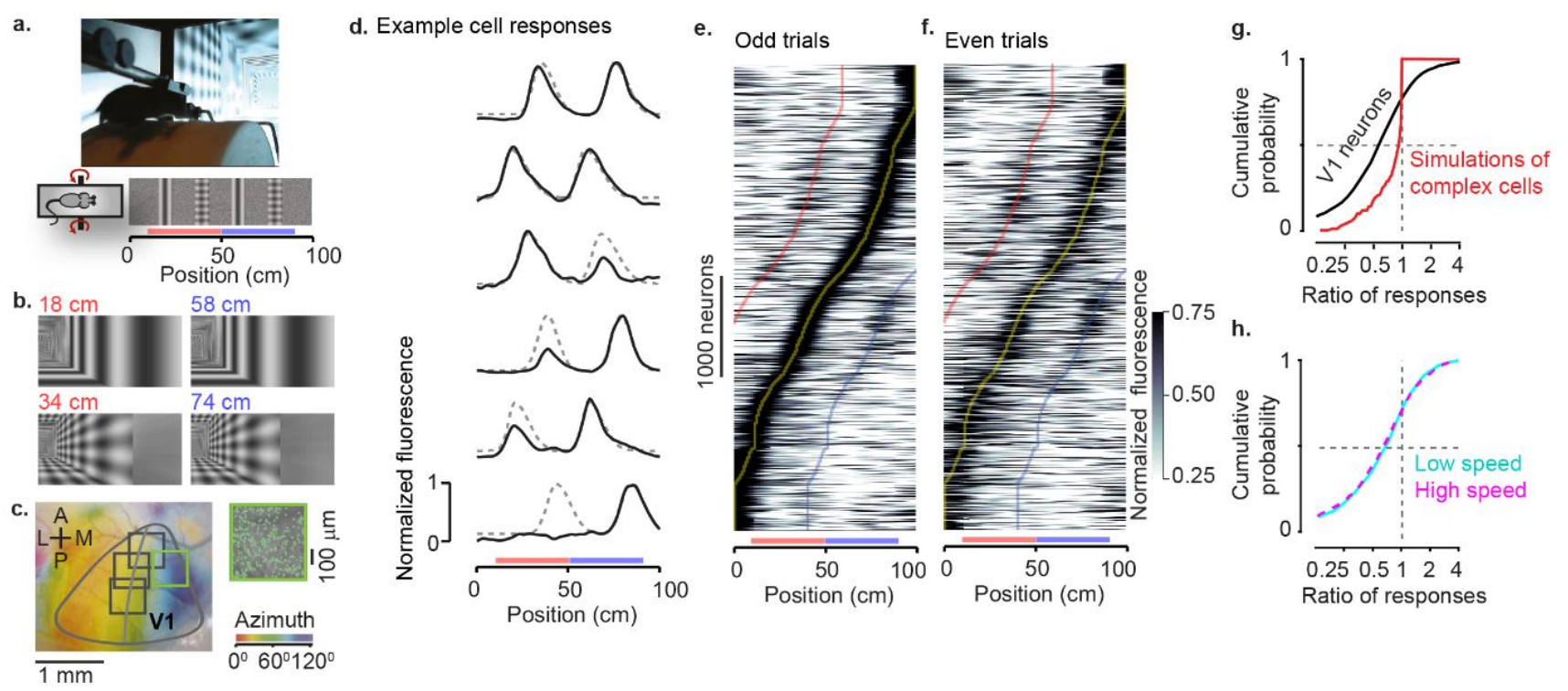

Figure 1: Responses in the primary visual cortex (V1) are modulated by spatial context. (a) Mice ran on a cylindrical treadmill to navigate a virtual corridor presented on three visual displays. The corridor had two landmarks (a grating and a plaid) that repeated after $40 \mathrm{~cm}$, creating two visually-matching segments (red and blue bars). (b) Example screenshots, showing the visual similarity of the virtual corridor at two pairs of positions spaced $40 \mathrm{~cm}$ apart. (c) Example retinotopic map of the cortical surface acquired with widefield calcium imaging. The border of V1 is defined by inversion of the retinotopic map. Squares denote the field of view in two-photon imaging sessions targeted to medial V1 in an animal (field of view with green frame is shown in the inset). Within these sessions we analysed responses from neurons with receptive field centre $>40^{\circ}$ azimuth (curve). (d) Normalized response as a function of position in the virtual environment for six example V1 neurons. Dotted lines show predictions if the responses were identical in the two segments of the corridor (red and blue bars). (e) Normalized response as a function of distance in the virtual environment obtained from odd trials only, for $\mathrm{V} 1$ neurons with receptive field centres $>40^{\circ}$ azimuth and activity significantly modulated by position in the corridor (4,958 of 8,610 neurons). Neurons are ordered based on the position of their maximum response. Yellow, red and blue lines indicate position of maximum $+/-40 \mathrm{~cm}$. Red and blue bars are as in a. (f) Same as e, for the half of the data (even trials) that were not used to order the responses. Sequence and scaling of the responses are the same as in e. (g) Cumulative distribution for the ratio of secondary response (40 cm away from peak response, red or blue line in $\mathrm{f}$ ) divided by peak response (yellow line in $\mathbf{f}$ ), derived from even trials (black). For comparison, the red curve shows the same ratio of responses obtained from simulations of Complex cells with purely visual responses. (h) Same as in $\mathbf{g}$ after stratifying the data by running speed. The two curves corresponding to low (cyan) and high (purple) speeds overlap and appear as a single dashed curve.

running speed or reward. As expected, applying a model of purely visual responses on the sequences of images (using a simulation of V1 complex cells, Supplementary Figure 3 ) generated ratios of responses that were very close to $1(0.97 \pm 0.17)$. The preference for spatial position could not be explained by deviations in pupil position or size as the ratio of responses was strongly biased towards low values even in sessions with steady eye position or pupil size (Supplementary Figure 4). Moreover, the modulation of $\mathrm{V} 1$ responses by spatial position could not be explained by variations in the animal's speed. Given that V1 neurons are influenced in diverse ways by running speed and visual speed ${ }^{9-12}$, they might respond differently in the two segments of the corridor based on speed differences. To control for this potential effect, we stratified the data in three groups according to instantaneous running speed (low, medium, or high; Supplementary Figure 5), and estimated each neuron's preferred position based on medium speed. We then calculated the ratio of responses for low and high speeds, and found them to be identical (Figure 1h). The spatial modulation of visual responses was also independent of the presence of a reward: in some sessions mice ran freely in the absence of a reward, and the ratio of responses remained significantly different from the simulations of purely visual responses (Supplementary Figure 6).

Having established that $\mathrm{V} 1$ responses are modulated by spatial position, we next asked whether the underlying signals reflect the spatial position encoded in the brain's navigational systems and the animal's subjective estimate of position (Figure 2). We 
recorded neuronal activity simultaneously in V1 and hippocampal area CA1 using two 32-channel extracellular electrodes in four wild-type mice (Figure 2a). To gauge their subjective estimate of position, we trained the mice to lick a water spout upon reaching a specific region of the VR corridor to receive a water reward (Figure 2b; Supplementary Movie 1, Supplementary Figure 7). All four mice learned to perform this task with over $80 \%$ accuracy. To ensure that the task required vision and could not be solved simply by counting steps, we introduced random intervals between consecutive runs through the corridor, and also tested that the animals could still perform the task when we changed the gain relating wheel rotation to progression in the VR corridor

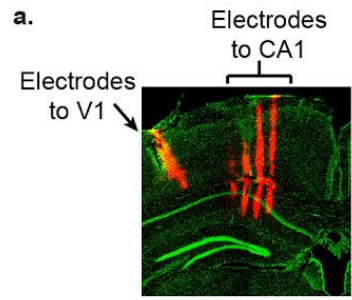

c.
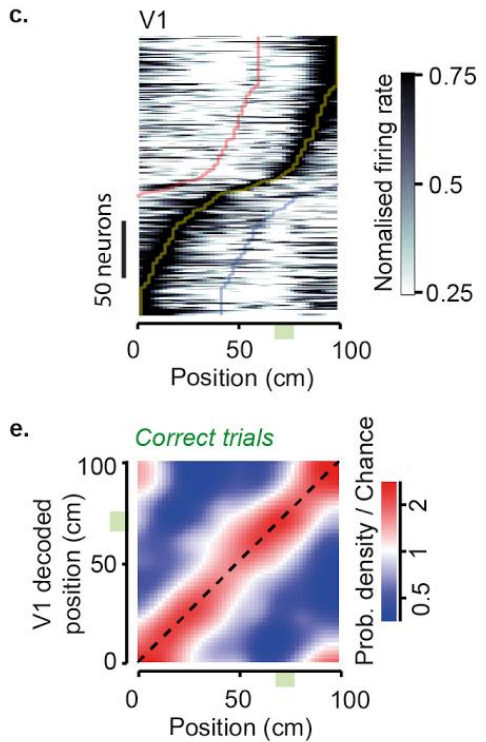

g. $\quad$ V1-CA1 error distributions

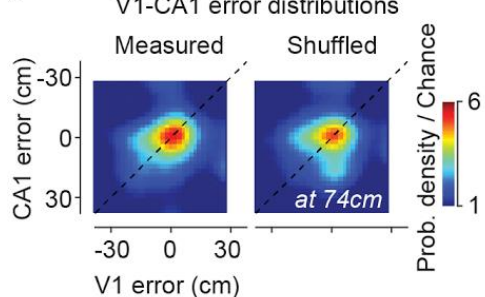

i.

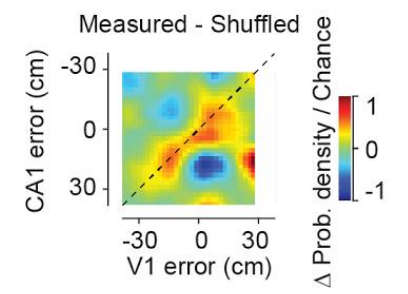

b.

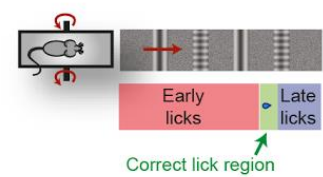

d.

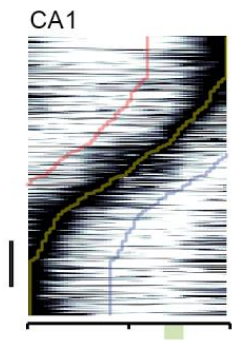

f.

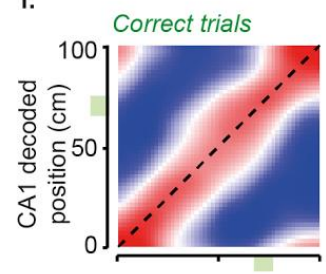

h.

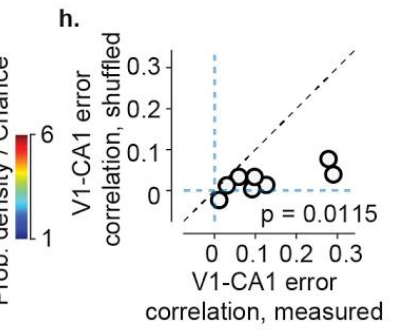

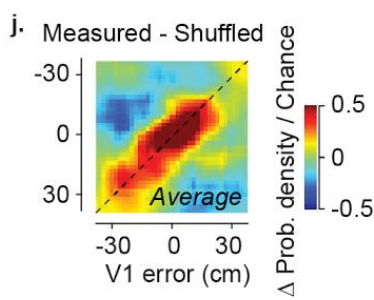

(Supplementary Fig $\mathbf{7 f , g}$ ). Moreover, performance was reduced when the contrast was lowered, thus confirming that the animal used vision to perform the task (Supplementary Figure 7c-e).

Many recorded neurons in both the visual cortex and hippocampus had place-specific response profiles and faithfully represented the position of the mouse in the environment (Figure 2c-f). Consistent with our previous observations with two-photon imaging, V1 neurons displayed a non-visual modulation of their response profiles, i.e. they responded more strongly in one of the two visually-matching segments of the corridor (Figure 2c). In turn, hippocampal CA1 neurons exhibited place fields ${ }^{4,13-15}$, responding in a single corridor location (Figure $2 \mathbf{d}$ ). Responses in both

Figure 2: V1 and CA1 neural populations represent spatial positions in the virtual corridor and make correlated errors. (a) Example of reconstructed electrode tracks. Multielectrode arrays were dipped in Dil before insertion (red); green shows cells labelled by DAPI. Panel shows one array (with four shanks) in the CA1 layer of the Hippocampus together with a second electrode track of one shank aimed at the primary visual cortex (V1). (b) In the task, water was delivered when mice licked in a reward zone (indicated by green shaded area in bottom of a). (c) Normalized activity as a function of position in the virtual corridor, for V1 neurons (266 cells, 8 sessions). Cells are ordered based on the position of their maximum response. Yellow, red and blue lines indicate position of maximum $+/-40 \mathrm{~cm}$. (d) Similar plot for CA1 place cells (334 cells, 8 sessions). (e) Density map showing the distribution of the position decoded from the firing of simultaneously recorded V1 neurons ( $y$-axis) as a function of the animal's actual position ( $x$-axis). The distribution of decoded positions was averaged across all recording sessions $(n=8)$, considering only correct trials. The red diagonal stripe indicates accurate estimation of position from the population. (f) Similar plot for CA1 neurons. (g) Density map showing the joint distribution of position decoding error from V1 and CA1 at an example position (left), together with a similar analysis on data shuffled preserving the correlation of run speed and position (right). (h) Correlation coefficient of decoding errors in V1 and CA1 for each recording session, against similar analysis of shuffled data. Correlations are significantly above shuffling control $(p=0.0198$, Student $t$ test). (i) Difference between density map for decoding errors from simultaneously recorded V1 and CA1 populations and shuffling control, for the example in $\mathbf{g}$. (j) Difference between joint density map of V1 and CA1 position, and shuffled control, averaged over all positions and recording sessions $(n=8)$. 
V1 and CA1 encoded the position of the mouse in the environment, with no ambiguity between the two visually-matching segments. Indeed, an independent Bayes decoder ${ }^{16}$ was able to read out the animal's position from the activity of neurons recorded from V1 ( $33 \pm 17$ neurons per session, $\mathrm{n}=8$ sessions; Figure 2e) or from CA1 ( $42 \pm 20$ neurons per sessions; $n=8$ sessions; Figure $2 \mathrm{f}$ ).

Furthermore, when visual cortex and hippocampus made errors in estimating the mouse's position, these errors were correlated with each other (Figure $\mathbf{2 g}$-h). The distributions of errors in position decoded from V1 and CA1 both peaked at zero (Figure $2 \mathrm{~g}$ ), but were significantly correlated (Figure $2 \mathrm{~h} ; \rho=0.125, \mathrm{p}=$ 0.0129 , Student t-test). Variations in speed affect responses of both $\mathrm{V} 1^{9,10}$ and $\mathrm{CA} 1^{16-19}$, so it is important to test whether this correlation arises from a common modulation of both regions by running speed. We therefore shuffled the data between trials in a manner that preserves the relationship between
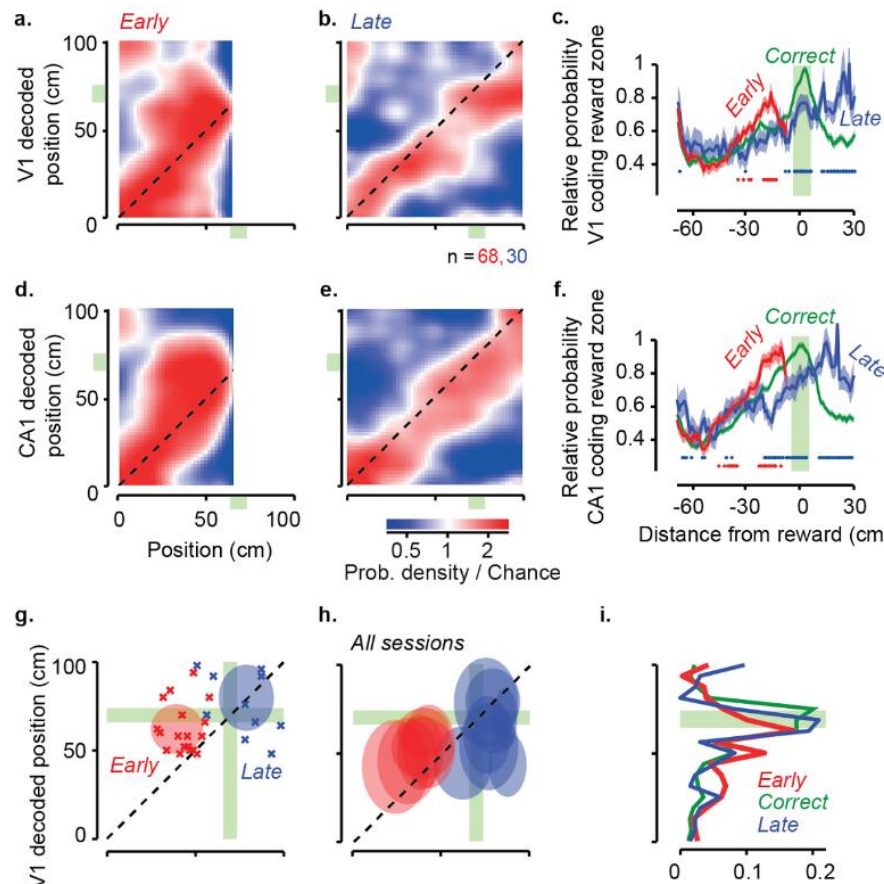

h.
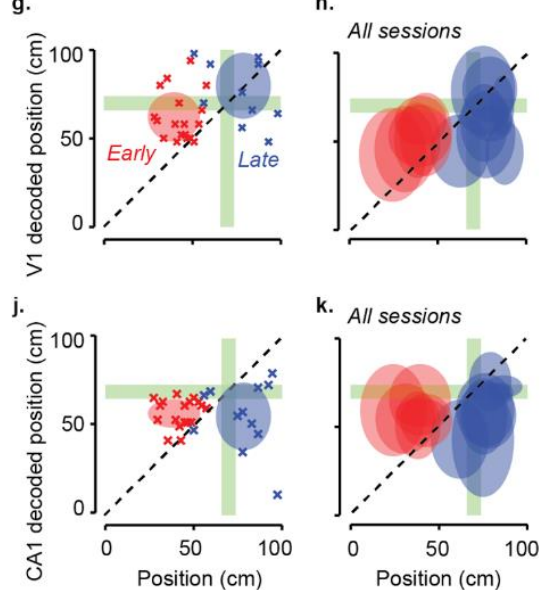

speed and position. After shuffling, the correlation dramatically decreased: V1 and CA1 decoding errors had a correlation of only 0.022 , significantly less than the observed moment-by-moment correlation of 0.125 ( $p=0.0115$; Figure $2 \mathrm{~g}, \mathrm{~h}$ ). To estimate how V1 and CA1 errors covary regardless of position and speed, we subtracted the joint distributions obtained from the original dataset and from the shuffled one (Figure $2 \mathbf{i}, \mathbf{j}$ ). The residual showed a clear distribution of decoding errors along the diagonal, indicating that V1 and CA1 representations are more correlated than expected from common speed modulation. Thus, on a moment-by-moment basis V1 carries an estimate of the animal's position that is consistent with the estimate in CA1.

We next asked whether the positional signals carried by CA1 and V1 relate to the animal's subjective estimate of position (Figure 3a-f). CA1 activity is influenced by the ongoing performance of navigation tasks ${ }^{20-23}$, and may reflect the animal's subjective

Figure 3: Positions encoded by visual cortex and hippocampus are correlated with animal's spatial decisions. (a) Density map showing the distribution of positions decoded from the V1 population ( $y$-axis), as a function of the animal's actual position (x-axis) on trials where the mice licked early. The decoder was trained on a separate set of trials where mice licked in the correct position. (b) Same plot for trials where the animal licked late. (c) The average decoded probability for the animal to be in the reward zone, as a function of distance from the reward on Early (red), Correct (green) and Late (blue) trials. The red curve peaks before the animal actually reaches the reward zone, while the blue curve peaks after, indicating that V1 population activity represents subjective, rather than actual position at the time of licking on early and late trials. The probability was normalized relative to the probability of being in the reward zone in the correct trials. Red dots: positions where the decoded probability of being in the reward zone differed significantly between Early and Correct trials ( $p<0.05$, two-sample t-test). Blue dots: significant difference between Correct and Late trials. (d-f) Same as a-c, for decoding using the population of CA1 neurons. (g) Position decoded from V1 activity as a function of actual position of the animal. Each cross shows the position of when the animal licked for a reward on Early trials (red crosses) or Late trials (blue crosses) on an example session. Note that Late trials can include some early licks. The distributions of all the error trial licks

during the example session (mean $\pm \mathrm{s} . \mathrm{d}$ ) are shown as shaded regions for Early (red) and Late (blue) trials. The reward zone is indicated as a light green shaded region. (h) The distributions of positions decoded from V1 activity as a function of actual position of the animal for all error trial licks across all recording sessions $(n=8)$. (i) Fraction of licks as a function of distance from reward location in decoded positions decoded from V1 activity. (j-I) Same as g-i, for CA1 neurons. 
position more than actual position ${ }^{21,23,24}$. Having trained the mice to selectively lick the spout in the reward zone, we could assess their subjective estimate of position from the location of their licks. We divided trials into three groups: Early trials when too many licks (usually 4-6) were before the reward zone, causing the trial to be aborted; Correct trials when one or more licks occurred in the reward zone; and Late trials when the mouse missed the reward zone and licked afterwards. To understand how V1 and CA1 neural representations of space related to this behaviour, we trained the Bayesian decoder on the population activity measured in Correct trials, and analysed the likelihood of decoding different positions in the three types of trials. Decoding performance in Early and Late trials showed systematic deviations from the diagonal (where decoded position is veridical). In Early trials, V1 and CA1 tended to overestimate the animal's progress along the corridor (a deviation above the diagonal in Figure $3 a, d)$. In Late trials, conversely, they tended to underestimate it (a deviation below the diagonal in Figure $3 \mathbf{b}, \mathbf{e})$. Accordingly, the probability of being in the reward zone peaked before the actual reward zone in Early trials and after it in Late trials, and this was the case not just for CA1 but also for V1 (Figure $3 c, f)$. These consistent deviations suggest that the representations of position in V1 and CA1 are correlated with the animal's decisions to lick and thus likely reflect the animal's subjective estimate of position.

The timing of the licks provides an opportunity to gauge when the mouse's subjective estimate of position lies in the reward zone. If activity in V1 and CA1 reflects subjective position, it should place the animal in the reward zone whether the animal correctly licked in that zone or it licked earlier or later. This prediction is borne out by the data (Figure $3 \mathrm{~g}-\mathrm{I}$ ). In the VR environment, we had a precise measurement of the times when the animal licked. We could therefore decode activity in V1 and CA1 at those times, and compare the decoded position to the animal's actual position. When plotted as a function of actual position, by definition, the distributions of licks in Early, Correct, and Late trials were distinct (Figure $\mathbf{3 g}, \mathbf{h}$ and $\mathbf{j}, \mathbf{k}$ ). However, when plotted as a function of decoded position (with a decoder trained only on Correct trials) these distributions came into register over the rewarded zone, whether the decoding was done from V1 activity (Figure $\mathbf{3 g}$-i) or from CA1 activity (Figure $\mathbf{3} \mathbf{j}$ -
I). Thus, when animals licked for a reward, the activity of both V1 and CA1 signalled that position was in the reward zone.

Taken together, these results indicate that the visual responses of $\mathrm{V} 1$ are modulated by the same spatial signals represented in hippocampus, and that these signals reflect the animal's subjective position in the environment. These signals may become stronger as animals become increasingly familiar with the environment ${ }^{6,7}$, perhaps contributing to the changes in $\mathrm{V} 1$ responses to visual stimuli seen as animals learn behavioural tasks ${ }^{25-27}$. However, we also observed spatial modulation in animals that freely ran the environment without reward (Figure 1; Supplementary Figure 6), suggesting that even incidental learning of the spatial features of the environment, in the absence of a task, is sufficient to modulate $\mathrm{V} 1$ responses.

The network of connections underlying this modulation of visual responses by subjective spatial position is yet unknown. While the primary visual cortex and hippocampus are not directly connected, both feed-forward and feedback connections (possibly through higher visual areas and retrosplenial or rhinal cortices) may convey spatial information between the two regions ${ }^{28,29}$. Outside the hippocampal formation, spatial signals have been reported in the retrosplenial, parietal and prefrontal corte $^{30,31}$. Our data show that spatial signals related to an animal's own estimate of position appear as early as in a primary sensory cortex. This result indicates that the mouse cortex does not keep a firm distinction between navigational and sensory systems: rather, spatial signals permeate cortical processing.

\section{Acknowledgements}

We thank Bilal Haider and other members of the lab for helpful discussions, and Neil Burgess for comments on the project. We thank Charu Reddy for help with histology, and Sylvia Schroeder and Michael Krumin with two-photon imaging. ABS is a recipient of the Sir Henry Dale Fellowship, awarded by Wellcome Trust / Royal Society (grant 200501). JF was supported by Human Frontier Science program (HFSP) and EC I Horizon 2020 (EU Framework Programme for Research and Innovation). MC and KDH are jointly funded by the Wellcome Trust (grant 095668 and 095669) and by the Simons Collaboration on the Global Brain (grant 325512). MC holds the 
GlaxoSmithKline/Fight for Sight Chair in Visual 14. Neuroscience.

\section{Author contributions}

All authors contributed to the design of the study. ABS \& MD carried out the experiments and analysed the data with JF. ABS, MD, JF, KDH and MC wrote the paper.

\section{References}

1. Wiener, S. I., Korshunov, V. A., Garcia, R. \& Berthoz, A. Inertial, Substratal and Landmark Cue Control of Hippocampal CA1 Place Cell Activity. Eur. J. Neurosci. 7, 2206-2219 (1995).

2. Muller, R. U. \& Kubie, J. L. The effects of changes in the environment on the spatial firing of hippocampal complex-spike cells. J. Neurosci. 7, 1951-1968 (1987).

3. Geiller, T., Fattahi, M., Choi, J.-S. \& Royer, S. Place cells are more strongly tied to landmarks in deep than in superficial CA1. Nat. Commun. 8, 14531 (2017).

4. Chen, G., King, J. A., Burgess, N. \& O’Keefe, J. How vision and movement combine in the hippocampal place code. Proc Natl Acad Sci U S A 110, 378-383 (2013).

5. Ji, D. \& Wilson, M. A. Coordinated memory replay in the visual cortex and hippocampus during sleep. Nat. Neurosci. 10, 100-107 (2007).

6. Haggerty, D. C. \& Ji, D. Activities of visual cortical and hippocampal neurons co-fluctuate in freely moving rats during spatial behavior. Elife 4, (2015).

7. Fiser, A. et al. Experience-dependent spatial expectations in mouse visual cortex. Nat. Neurosci. 19, 1658-1664 (2016).

8. Kalatsky, V. A. \& Stryker, M. P. New paradigm for optical imaging: Temporally encoded maps of intrinsic signal. Neuron 38, 529-545 (2003).

9. Niell, C. M. \& Stryker, M. P. Modulation of Visual Responses by Behavioral State in Mouse Visual Cortex. Neuron 65, 472-479 (2010).

10. Saleem, A. B., Ayaz, A., Jeffery, K. J., Harris, K. D. \& Carandini, M. Integration of visual motion and locomotion in mouse visual cortex. Nat. Neurosci. 16, 1864-1869 (2013).

11. Ayaz, A., Saleem, A. B., Schölvinck, M. L. \& Carandini, M. Locomotion controls spatial integration in mouse visual cortex. Curr. Biol. 23, 890-894 (2013).

12. Keller, G. B., Bonhoeffer, T. \& Hubener, M. Sensorimotor mismatch signals in primary visual cortex of the behaving mouse. Neuron 74, 809815 (2012).

13. Ravassard, P. et al. Multisensory Control of Hippocampal Spatiotemporal Selectivity. Science 340, 1342-1346 (2013).
14. Dombeck, D. A., Harvey, C. D., Tian, L., Looger, L. L. \& Tank, D. W. Functional imaging of hippocampal place cells at cellular resolution during virtual navigation. Nat. Neurosci. 13, 1433-1440 (2010).

15. Harvey, C. D., Collman, F., Dombeck, D. A. \& Tank, D. W. Intracellular dynamics of hippocampal place cells during virtual navigation. Nature 461, 941946 (2009).

16. Zhang, K., Ginzburg, I., McNaughton, B. L. \& Sejnowski, T. J. Interpreting neuronal population activity by reconstruction: unified framework with application to hippocampal place cells. J. Neurophysiol. 79, 1017-44 (1998).

17. Wiener, S. I., Paul, C. A. \& Eichenbaum, H. B. Spatial and behavioral correlates of hippocampal neuronal activity. J. Neurosci. 9, 2737-63 (1989).

18. Czurkó, A., Hirase, H., Csicsvari, J. \& Buzsáki, G. Sustained activation of hippocampal pyramidal cells by 'space clamping' in a running wheel. Eur. J. Neurosci. 11, 344-352 (1999).

19. McNaughton, B. L., Barnes, C. A. \& O'Keefe, J. The contributions of position, direction, and velocity to single unit activity in the hippocampus of freelymoving rats. Exp. Brain Res. 52, 41-49 (1983).

20. Hok, V. et al. Goal-related activity in hippocampal place cells. J.Neurosci. 27, 472-482 (2007).

21. Lenck-Santini, P.-P., Muller, R. U., Save, E. \& Poucet, B. Relationships between place cell firing fields and navigational decisions by rats. J. Neurosci. 22, 9035-9047 (2002).

22. Lenck-Santini, P. P., Save, E. \& Poucet, B. Evidence for a relationship between place-cell spatial firing and spatial memory performance. Hippocampus 11, 377-390 (2001).

23. O'Keefe, J. \& Speakman, A. Single unit activity in the rat hippocampus during a spatial memory task. Exp Brain Res 68, 1-27 (1987).

24. Rosenzweig, E. S., Redish, A. D., McNaughton, B. L. \& Barnes, C. A. Hippocampal map realignment and spatial learning. Nat. Neurosci. 6, 609-615 (2003).

25. Jurjut, O., Georgieva, P., Busse, L. \& Katzner, S. Learning Enhances Sensory Processing in Mouse V1 before Improving Behavior. J. Neurosci. 37, 6460-6474 (2017).

26. Poort, J. et al. Learning Enhances Sensory and Multiple Non-sensory Representations in Primary Visual Cortex. Neuron 86, 1478-1490 (2015).

27. Makino, H. \& Komiyama, T. Learning enhances the relative impact of top-down processing in the visual cortex. Nat. Neurosci. 18, 1116-1122 (2015).

28. Wang, Q., Gao, E. \& Burkhalter, A. Gateways of Ventral and Dorsal Streams in Mouse Visual Cortex. J. Neurosci. 31, 1905-1918 (2011).

29. Witter, M. P. et al. Cortico-hippocampal communication by way of parallel parahippocampal-subicular pathways. 
bioRxiv preprint doi: https://doi.org/10.1101/235648; this version posted December 18, 2017. The copyright holder for this preprint (which was not certified by peer review) is the author/funder, who has granted bioRxiv a license to display the preprint in perpetuity. It is made available under aCC-BY-NC-ND 4.0 International license.

Hippocampus 10, 398-410 (2000).

30. Grieves, R. M. \& Jeffery, K. J. The representation of space in the brain. Behavioural Processes 135, 113-131 (2017).
31. Moser, E. I., Kropff, E. \& Moser, M.-B. Place Cells, Grid Cells, and the Brain's Spatial Representation System. Annu. Rev. Neurosci. 31, 69-89 (2008). 


\section{Supplementary Figures}

Supplementary Movie 1: Movie of animal performing the task in virtual reality.

a.
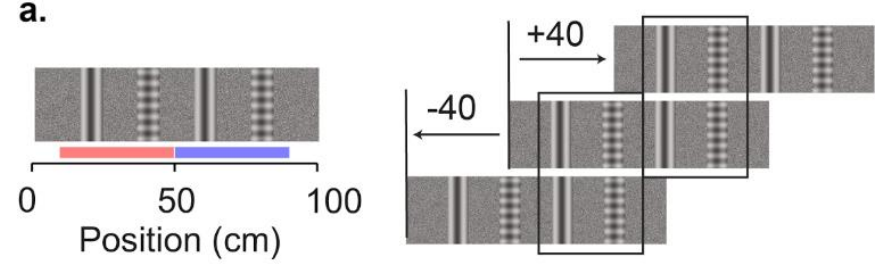

b. Images over right visual field at positions:
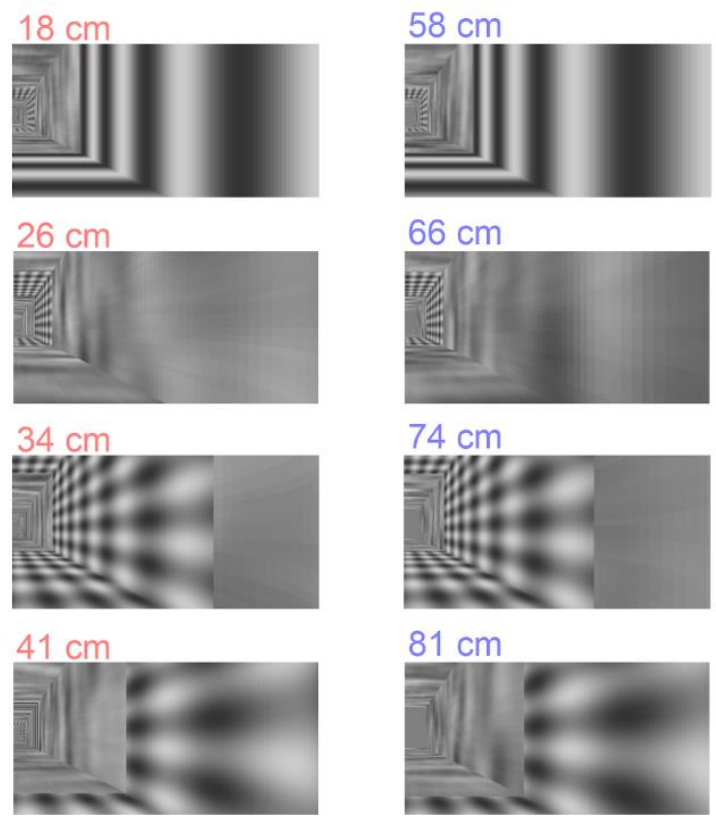

Supplementary Figure 1: Design of virtual environment with two visually matching segments.

a, The virtual corridor had four prominent landmarks. Two landmarks (grating and plaid) were repeated at two positions, $40 \mathrm{~cm}$ apart creating two visually matching segments in the room, from $10 \mathrm{~cm}$ to $50 \mathrm{~cm}$ and from 50 $\mathrm{cm}$ to $90 \mathrm{~cm}$ (indicated by red and blue bars in the left panel), as illustrated in the right panel.

b, Example screenshots of the right visual field displayed in the environment when the animal is at different positions. Each row displays screen images at positions $40 \mathrm{~cm}$ apart. 
a.

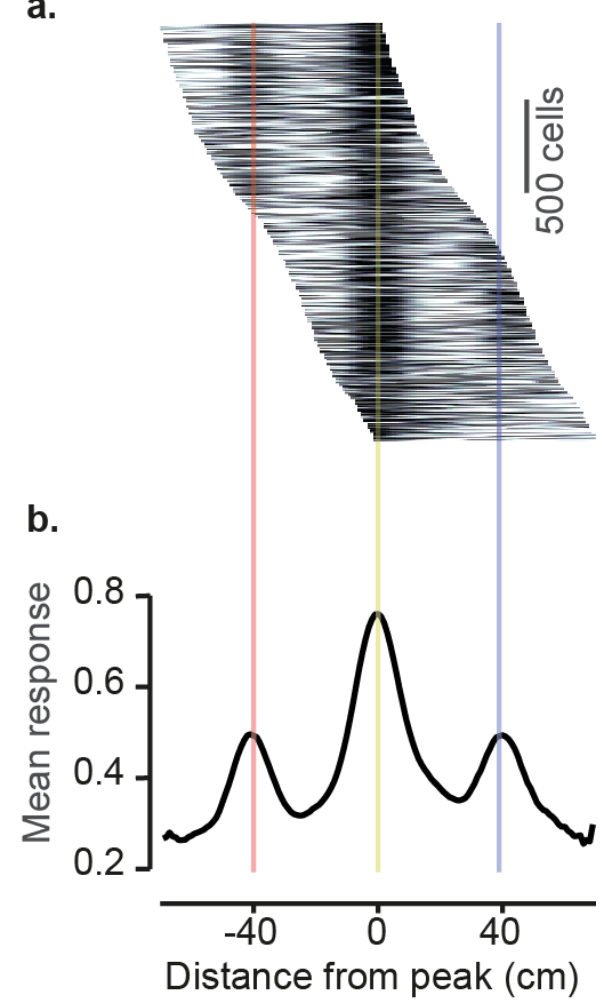

Supplementary Figure 2: Spatial averaging of visual cortical activity confirms the difference in response between visually matching locations.

a, Mean response of $\mathrm{V} 1$ neurons as a function of the distance to the peak response, as obtained from even trials (2,422 cells with peak response between 15 and $85 \mathrm{~cm}$ along the corridor). The position of the peak response was estimated from the other half of the trials (odd trials).

b, Population average across responses shown in a. Lower values of the side peaks compared to central peak indicates strong preference of V1 neurons for one segment of the corridor over the other visually-matching segment (40 $\mathrm{cm}$ away from peak response) . 


\section{Complex cell simulations}

a. Spatial Freq: $0.04 \mathrm{cyc} /{ }^{\circ}$

RF Centre: $60^{\circ}$

Orientation: $0^{\circ}$
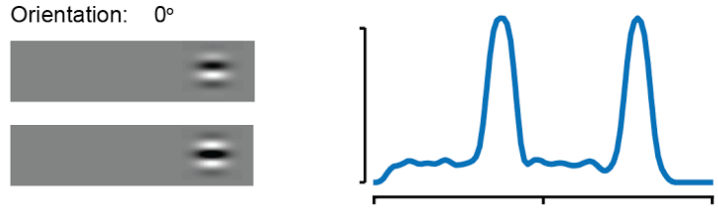

c. Spatial Freq: $0.07 \mathrm{cyc} /{ }^{\circ}$

RF Centre: $30^{\circ}$

Orientation: 0

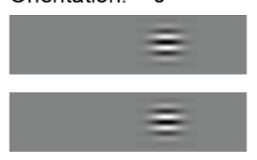

e. Spatial Freq: $0.04 \mathrm{cyc} /{ }^{\circ}$

RF Centre: $30^{\circ}$

Orientation: $60^{\circ}$
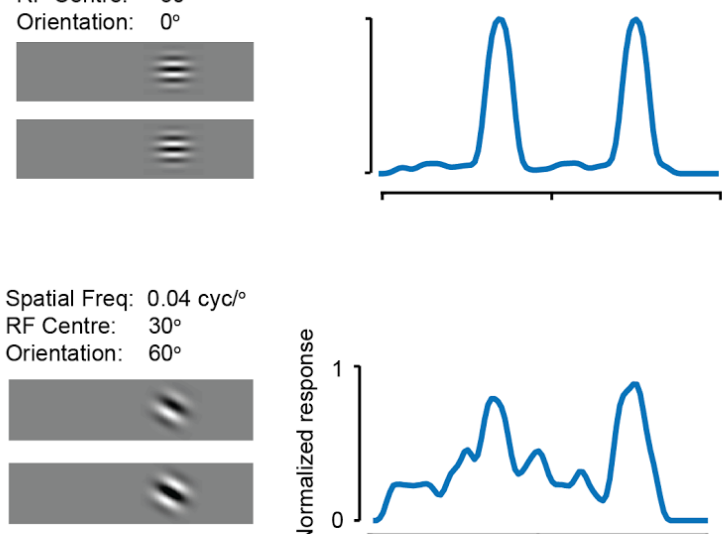

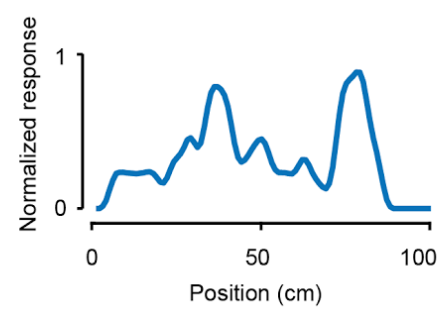

b. Spatial Freq: $0.04 \mathrm{cyc} /{ }^{\circ}$

RF Centre: $30^{\circ}$

Orientation: $0^{\circ}$
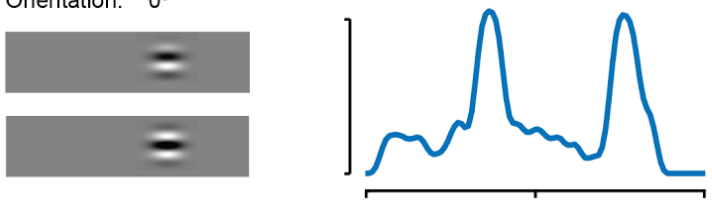

d.

Spatial Freq: $0.04 \mathrm{cyc} /{ }^{\circ}$

RF Centre: $30^{\circ}$

Orientation: $90^{\circ}$
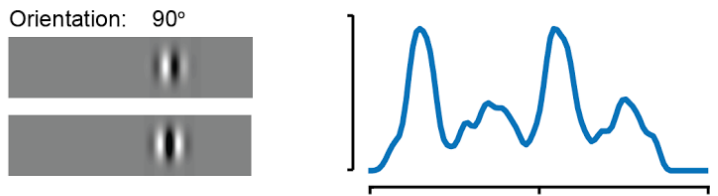

f. Spatial Freq: $0.04 \mathrm{cyc} /{ }^{\circ}$

RF Centre: $30^{\circ}$

Orientation: $135^{\circ}$
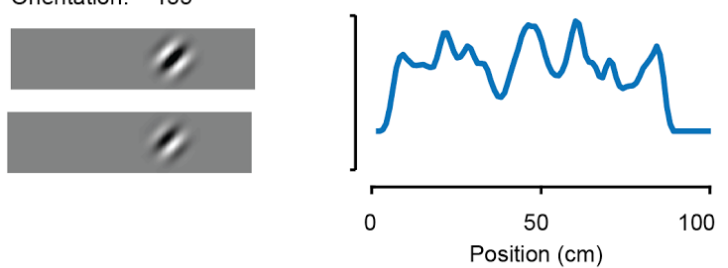

\section{Supplementary Figure 3: Simulation of purely visual responses to position in VR.}

Responses of 6 simulated neurons, with purely visual responses produced by a complex cell model, with varying spatial frequency, orientation, or receptive field centres. The images on the left of the panel show the quadrature pair of complex cell filters, and on the right is the response of the simulation of that pair as a function of position in the virtual environment. Simulated complex cells had spatial frequencies, and orientations that are commonly observed in mouse V1 (sf: $0.04,0.05,0.06$ or $0.07 \mathrm{cyc} /{ }^{\circ}$; orientations: $0^{\circ}$ to $90^{\circ}$ with twice more cells for cardinal orientations). The receptive field had positions $>40^{\circ}$ azimuth $\left(40^{\circ}, 50^{\circ}\right.$, $70^{\circ}, 80^{\circ}$ ), similar to the V1 neurons we considered for analysis. In rare cases (like in $\mathrm{f}$ ) when the receptive fields do not match the features of the environment, we get little selectivity along the corridor. These cases lead to lower value for the response ratios. 


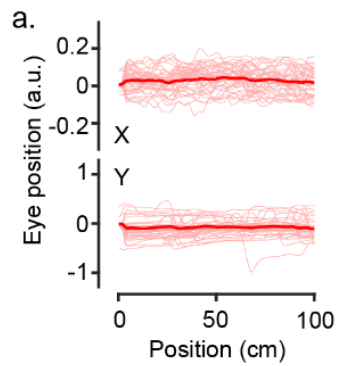

C.

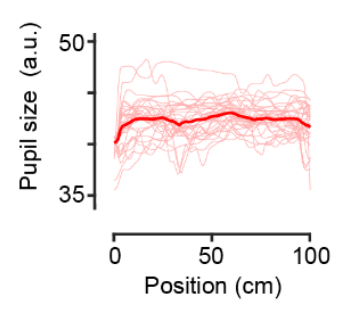

b.

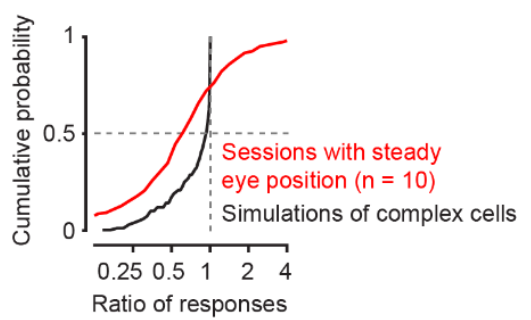

d.

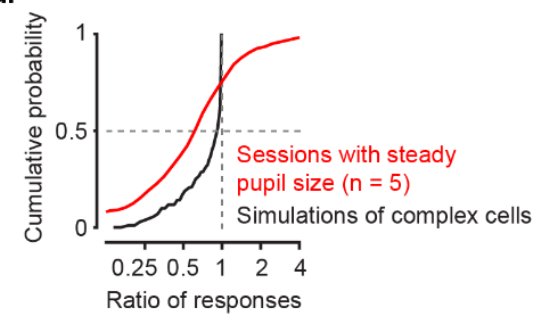

Supplementary Figure 4: The spatial modulation of $\mathrm{V} 1$ responses is not a result of pupil position or diameter.

a, Pupil position as a function of location in the virtual corridor, for an example session where the position of the pupil was on average the same along the corridor. Thin red curves: position trajectories on individual trials; thick curves, average. Top and bottom panels: $x$ - and $y$-coordinates of the pupil.

b, Distribution of response ratios for sessions with steady eye position (10 sessions; red) and for simulations of complex cells (black).The two distributions are significantly different (two-sample Kolmogorov-Smirnov test; $p<<0.001)$.

c, Pupil size as a function of position for an example session where pupil size was on average the same along the VR corridor.

d, Distribution of response ratios for sessions with steady pupil size ( 5 sessions; red) and for simulations of complex cells (black). The two distributions are significantly different (two-sample Kolmogorov-Smirnov test; $\mathrm{p}<<0.001)$. 
a.

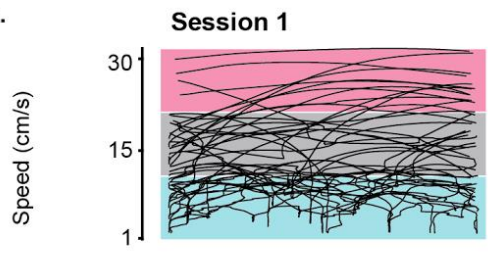

b.

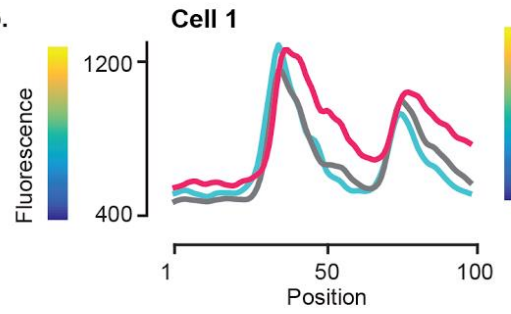

c.

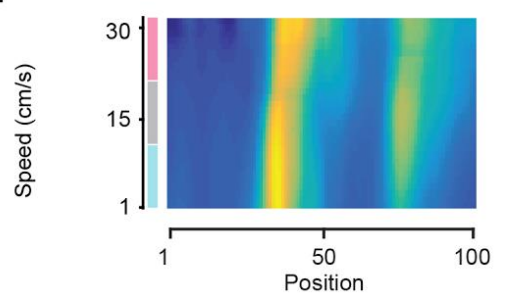

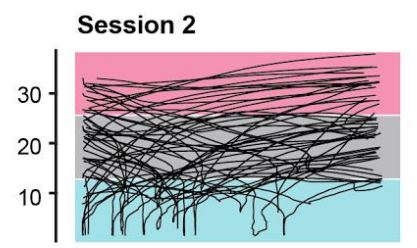
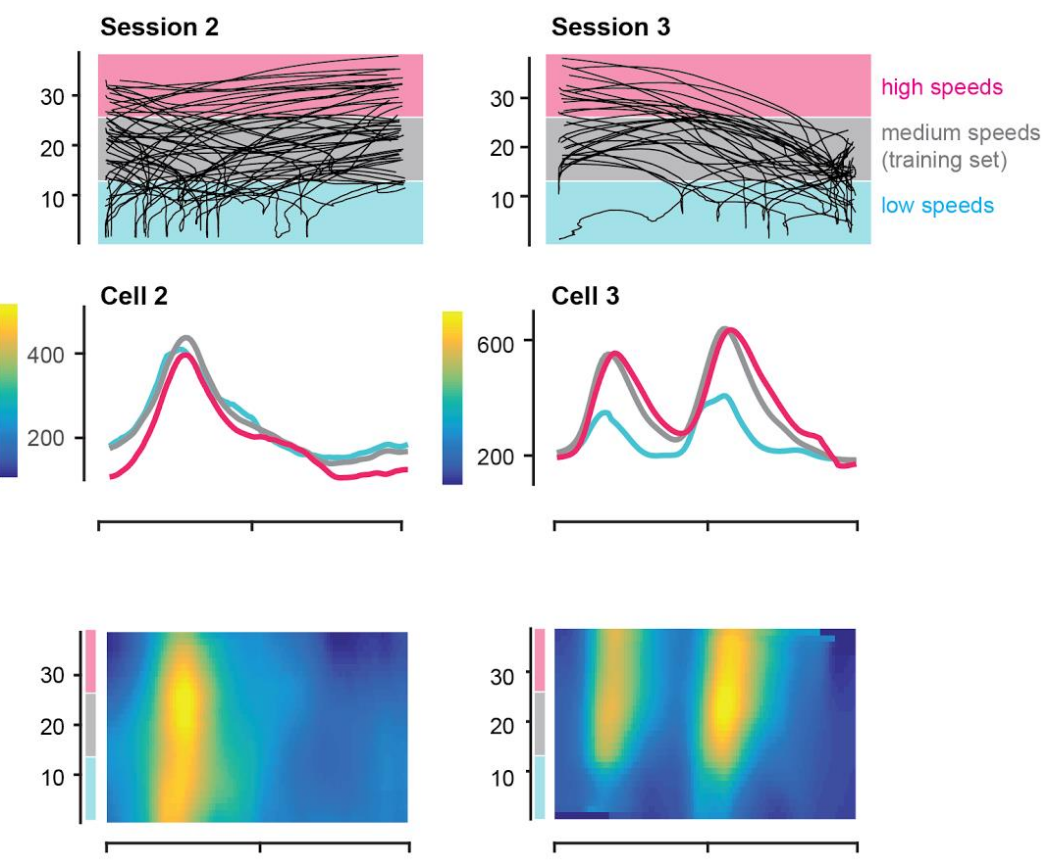

Supplementary Figure 5: The spatial modulation of responses in V1 cannot be explained by speed.

a, Single-trial trajectories of speed as a function of position in the virtual reality environment for three example recording sessions.

b, Response profile of an example $\mathrm{V} 1$ cells in each session as a function of position in the room for three different speed ranges, corresponding to the 3 shading bands in a.

c, Two-dimensional response profiles of the same example neurons showing activity as a function of position and running speed for speeds higher than $1 \mathrm{~cm} / \mathrm{s}$. 
a. Odd trials

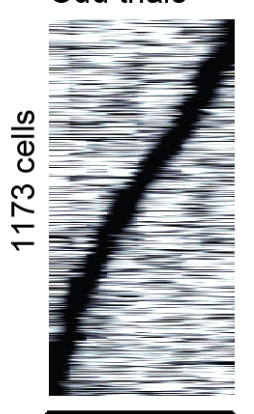

150100

Position (cm)
Even trials

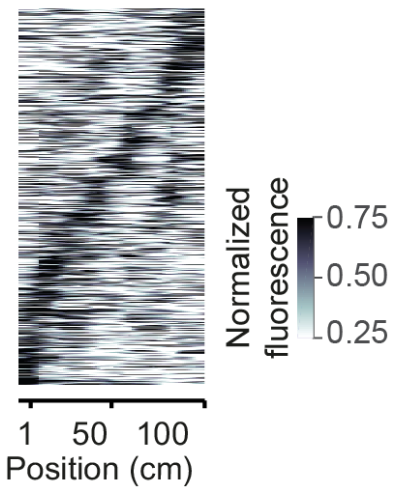

b.

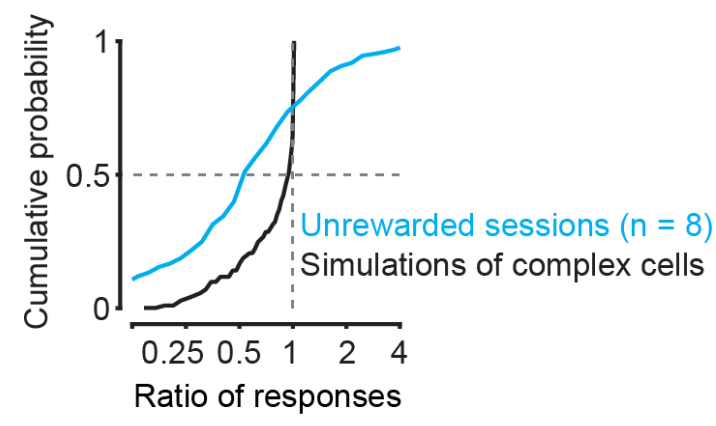

Supplementary Figure 6: The spatial modulation of V1 responses cannot be explained by reward.

a, Normalized response as a function of position in the virtual corridor, across sessions without reward (1173 cells). Data come from 2 out 4 mice that freely ran the environment without reward ( 8 sessions). Responses in even trials (right) are ordered according to the position of maximum activity measured in odd trials (left).

b, Distribution of response ratios for unrewarded sessions (8 sessions; cyan) and for simulations of complex cells (black). The two distributions are significantly different (two-sample Kolmogorov-Smirnov test; $\mathrm{p}<<0.001)$. 
a.

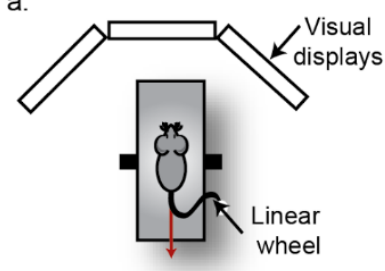

b.

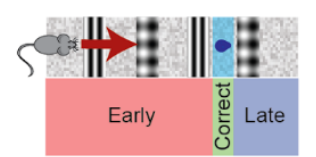

C.

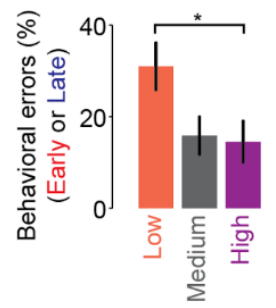

d.

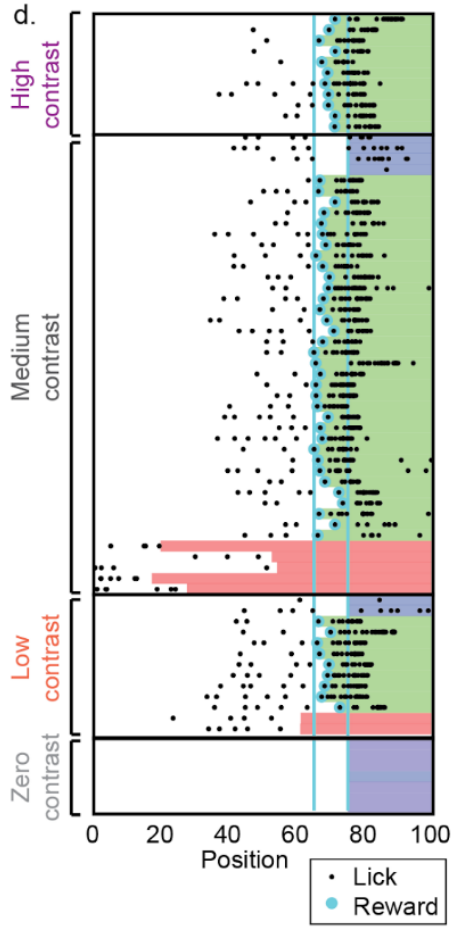

e.

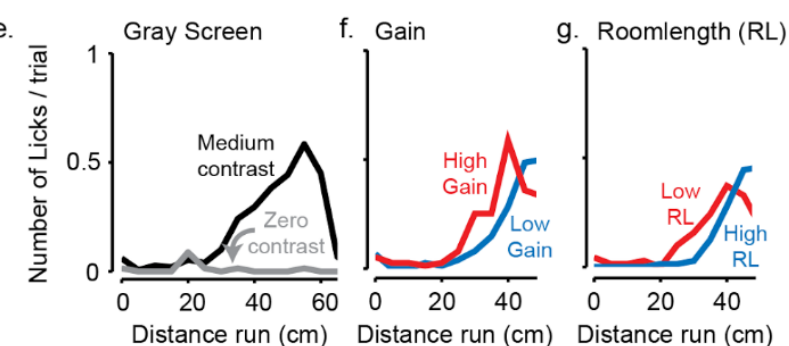

Supplementary Figure 7: Behavioural performance in the task.

a, Cartoon of the experimental setup. Virtual reality was presented on three visual displays.

b, Illustration of the virtual reality environment with four prominent landmarks, a reward zone, and the zones that define trial types: Early, Correct and Late.

c, Percentage of trials during which the animal makes behavioural errors, by licking either too early or too late at three different contrast levels: $18 \%$ (low), 60\% (medium) or $72 \%$ (high).

d, Illustration of performance on all trials of one example recording session. Each row represents a trial, black dots represent positions where the animal licked, and cyan dots indicate the delivery of a water reward. Coloured bars indicate the outcome of the trial, red: Early, green: Correct, blue: Late.

e-g, Successful performance relies on vision: e, The animal does not lick when the room was presented at zero contrast. f. If the gain between the animals' physical movement and movement in the virtual environment was increased, the animals licked after running a shorter physical distance. $\mathbf{g}$, If the position of the visual cues was shifted forward or back (high/low room length $(\mathrm{RL})$ ), the lick position shifted accordingly, indicating that the animals rely on vision to perform the task. 


\section{Methods}

All experiments were conducted according to the UK Animals (Scientific Procedures) Act, 1986 under personal and project licenses issued by the Home Office following ethical review.

For simultaneous recordings in V1 and CA1, we used four C57BL/6 mice (all male, implanted at 4-8 weeks of age). For calcium imaging experiments, we used double or triple transgenic mice expressing GCaMP6 in excitatory neurons ( 3 females, 1 male, implanted at 4-6 weeks). The double transgenic expressed GCaMP6 slow (Wekselblatt et al., 2016) (Camk2a-tTA;tetO-G6s). The triple transgenics expressed GCaMP6 fast (Madisen et al., 2015) (Emx1- Cre;Camk2a-tTA;Ai93, 3 mice). None of these mice displayed the aberrant activity that is sometimes seen in Ai93 mice (Steinmetz et al., 2017).

\section{Virtual Environment and task}

The virtual reality environment was a corridor adorned with a white noise background and four landmarks: two grating stimuli oriented orthogonal to the corridor and two plaid stimuli (Figure 1a). The corridor dimensions were $100 \times 8 \times 8 \mathrm{~cm}$, and the landmarks ( $8 \mathrm{~cm}$ wide) were centred $20,40,60$ and $80 \mathrm{~cm}$ from the start of the corridor. The animal navigated the environment by walking on a custom-made polystyrene wheel (15 cm wide, $18 \mathrm{~cm}$ diameter). Movements of the wheel were captured by a rotary encoder (2400 pulses/rotation, Kübler, Germany), and used to control the virtual reality environment presented on three monitors surrounding the animal, as previously described(Saleem et al., 2013). When the animal reached the end of the corridor, it was placed back at the start of the corridor after a 3-5 s presentation of a grey screen. Trials longer than $120 \mathrm{~s}$ were timed out and were excluded from further analysis.

Mice used for calcium imaging ran freely through the corridor, with no specific task. Two of the mice were motivated to run with water rewards. One animal received rewards at random positions along the corridor. The other received rewards at the end of the corridor. To control for the effect of the reward on V1 responses, no reward was delivered in some sessions ( $\mathrm{n}=8$ sessions; 2 animals).

Mice used for simultaneous V1-CA1 recordings were trained to lick in a specific region of the corridor, the reward zone. This zone was centred at $70 \mathrm{~cm}$ and was $8 \mathrm{~cm}$ wide. Trials in which the animals were not engaged in the task, i.e. when they ran through the environment without licking, were excluded from further analysis. The animal was rewarded for correct licks with $\sim 2 \mu$ l water using a solenoid valve (161T010; Neptune Research, USA), and licks were monitored using a custom device that detected breaks in an infrared beam.

\section{Surgery and training}

The surgical methods are similar to those described previously (Ayaz et al., 2013; Saleem et al., 2013). Briefly, a custom head-plate with a circular chamber (3-4 mm diameter for electrophysiology; $8 \mathrm{~mm}$ for imaging) was implanted on 4-8 week mice under isoflurane anaesthesia. For imaging, we performed a $4 \mathrm{~mm}$ craniotomy over left visual cortex by repeatedly rotating a biopsy punch. The craniotomy was shielded with a double coverslip ( $4 \mathrm{~mm}$ inner diameter; $5 \mathrm{~mm}$ outer diameter). After 4 days of recovery, some mice were water restricted (> $40 \mathrm{ml} / \mathrm{kg} /$ day) and were trained for 30-60 $\mathrm{min}$, 5-7 days/week.

Mice used for simultaneous V1-CA1 recordings were trained to lick selectively in the reward zone using a progressive training procedure. Initially, the animals were rewarded for running past the reward location on all trials. After this, we introduced trials where the animal was rewarded only when it licked in the rewarded region of the corridor. The width of the reward region was progressively narrowed from $30 \mathrm{~cm}$ to $8 \mathrm{~cm}$ across successive days of training. To prevent the animals from licking all across the corridor, trials were terminated early if the animal licked more than a certain number of times before the rewarded region. We reduced this number as the animals performed more accurately, typically reaching a level of 4-6 licks by the time recordings were made. Once a sufficient level of performance was reached, we controlled on some (random) trials that the animal performed the task visually by measuring the performance when decreasing the visual contrast or changing the distance to the reward zone (Supplementary Figure 7). Training was carried out for 3-5 weeks. Animals were light shifted ( 9 am light off, 9 pm light on) and experiments were performed during the day. 


\section{Widefield calcium imaging}

For widefield imaging we used a standard epi-illumination imaging system (Ratzlaff and Grinvald, 1991; Carandini et al., 2015) together with an SCMOS camera (pco.edge, PCO AG). A Leica 1.6x Plan APO objective was placed above the imaging window and a custom black cone surrounding the objective was fixed on top of the headplate to prevent contamination from the monitors' light. The excitation light beam emitted by a highpower LED (465 nm LEX2-B, Brain Vision) was directed onto the imaging window by a dichroic mirror designed to reflect blue light. Emitted fluorescence passed through the same dichroic mirror and was then selectively transmitted by an emission filter (FF01-543/50-25, Semrock) before being focused by another objective (Leica 1.0 Plan APO objective) and finally detected by the camera. Images of $200 \times 180$ pixels, corresponding to an area of $6.0 \times 5.4 \mathrm{~mm}$ were acquired at $50 \mathrm{~Hz}$.

To measure retinotopy we presented a $14^{\circ}$-wide vertical window containing a vertical grating (spatial frequency 0.15 cycles/deg), and swept (Kalatsky and Stryker, 2003; Yang et al., 2007) the horizontal position of the window over $135^{\circ}$ of azimuth angle, at a frequency of $2 \mathrm{~Hz}$. Stimuli lasted $4 \mathrm{~s}$ and were repeated 20 times (10 in each direction). We obtained maps for preferred azimuth by combining responses to the 2 stimuli moving in opposite direction, as previously described (Kalatsky and Stryker, 2003).

\section{Two-photon imaging}

Two-photon imaging was performed with a standard multiphoton imaging system (Bergamo II; Thorlabs) controlled by Scanlmage4 (Pologruto et al., 2003). A $970 \mathrm{~nm}$ laser beam, emitted by a Ti:Sapphire Laser (Chameleon Vision, Coherent), was targeted onto L2/3 neurons through a $16 \mathrm{x}$ water-immersion objective $(0.8$ NA, Nikon). Fluorescence signal was transmitted by a dichroic beamsplitter and amplified by photomultiplier tubes (GaAsP, Hamamatsu). The emission light path between the focal plane and the objective was shielded with a custom-made plastic cone, to prevent contamination from the monitors' light. In each experiment, we imaged 4 planes set apart by $40 \mu \mathrm{m}$. Multiple-plane imaging was enabled by a piezo focusing device (P-725.4CA PIFOC, Physik Instrumente), and an electro-optical modulator (M350-80LA, Conoptics Inc.) which allowed adjustment of the laser power with depth. Images of $512 \times 512$ pixels, corresponding to a field of view of $500 \times 500 \mu \mathrm{m}$, were acquired at a frame rate of $30 \mathrm{~Hz}(7.5 \mathrm{~Hz}$ per plane).

Pre-processing of raw imaging movies involved 1) image registration to correct for brain movement, 2) ROI extraction, i.e. cell detection and 3) correction for neuropil contamination. Image registration, cell detection and neuropil signal extraction were performed with the Suite2p pipeline (Pachitariu et al., 2016). Cells with noisy baseline or extremely seldom firing were excluded from further analysis.

For neuropil correction, we used an established method (Peron et al., 2015; Dipoppa et al., 2016). We used Suite $2 p$ to determine a mask surrounding each cell's soma, the 'neuropil mask'. The inner diameter of the mask was $3 \mu \mathrm{m}$ and the outer diameter was $<45 \mu \mathrm{m}$. For each cell we obtained a correction factor, $\alpha$, by regressing the binned neuropil signal ( 20 bins in total) from the $5^{\text {th }}$ percentile of the raw binned cell signal. For a given session, we obtained the average correction factor across cells. This average factor was used to obtain the corrected individual cell traces, from the raw cell traces and the neuropil signal, assuming a linear relationship. All correction factors fell within 0.7 and 0.9 .

\section{Pupil tracking}

We tracked the eye of the animal using an infrared camera (DMK 21BU04.H, Imaging Source) and a zoom lens (MVL7000, Navitar) at $25 \mathrm{~Hz}$. Pupil position and size were calculated by fitting an ellipsoid to the pupil for each frame using a custom software. $X$ and $Y$ positions of the pupil were derived from the centre of mass of the fitted ellipsoid.

\section{Electrophysiological recordings}

On the day prior to the first recording session, we made two $1 \mathrm{~mm}$ craniotomies, one over CA1 $(1.0 \mathrm{~mm}$ lateral, $2.0 \mathrm{~mm}$ anterior from lambda), and a second one over V1 ( $2.5 \mathrm{~mm}$ lateral, $0.5 \mathrm{~mm}$ anterior from lambda). We covered the chamber using KwikCast (World Precision Instruments) and the animals were allowed to recover overnight. The CA1 probe was lowered until all shanks were in the pyramidal layer. We waited $\sim 30 \mathrm{~min}$ for the tissue to settle before starting the recordings. In two animals, we dipped the probes in red-fluorescent Dil (Figure 2a). In these animals, we had only one recording session. The other animals had two and four recording sessions. 
Offline spike sorting was carried out using the KlustaSuite (Rossant et al., 2016) package, with automated spike sorting using KlustaKwik (Kadir et al., 2014), followed by manual refinement using KlustaViewa (Rossant et al., 2016). Hippocampal interneurons were identified based on their spike time autocorrelation and excluded from further analysis. Only time points with running speeds greater than $5 \mathrm{~cm} / \mathrm{s}$ were included in further analyses.

\section{Analysis of response profiles for electrophysiological data}

To calculate the response profile of each neuron as a function of position in the virtual corridor we used a local smoothing method (Loader, 1996; Harris et al., 2002, 2003). We first smoothed the firing rate using a $250 \mathrm{~ms}$ Gaussian window. We then discretized the position of the animal in $2 \mathrm{~cm}$ bins, yielding 50 bins and we calculated the spike count map and occupancy map for each neuron. Both the spike count and occupancy maps were smoothed by convolving them with a common Gaussian window whose width was optimized to maximize reliability (see below), and the response profile was calculated as the ratio of the smoothed spike count map and the occupancy map (Saleem et al., 2013).

Response profile reliability was calculated as the fraction of variance in firing rate explained by the response profile:

$$
\text { Reliability }=1-\frac{\sum_{t}\left(y(t)-y^{\prime}(t)\right)^{2}}{\sum_{t}(y(t)-\mu)^{2}}
$$

where $y(t)$ is the firing rate of the neuron at time $t, y^{\prime}(t)$ was the prediction by the place-field for the same time bin and $\mu$ is the mean firing rate of the training data(Saleem et al., 2013). We used five-fold cross-validation to calculate place fields and reliability. Only neurons with a reliability greater than 0.01 were considered for further analysis.

\section{Analysis of response profiles for two-photon data}

To obtain response profiles as a function of position along the corridor, we averaged the neuropil-corrected activity of each cell in 1-cm-wide bins (100 bins in total) and smoothed with a $5 \mathrm{~cm}$ Gaussian window. Only time points with running speeds greater than $1 \mathrm{~cm} / \mathrm{s}$ were included in further analyses. For consistency with the response profiles obtained from electrophysiological data, we only looked at responses for which the cross-validated reliability was higher than 0.01 . These cells were considered to have activity significantly modulated by position in the corridor. To model single-cell activity under the assumption that responses are identical in the two segments of the corridor, we fit (using least squares) a model function to the response profile along the visually-matching segment where the cell peaked. The model function was the sum of two Gaussians that meet at the peak. To obtain a prediction along the whole corridor, we then duplicated the fitted response at $\pm 40 \mathrm{~cm}$ away from the maximum. Cells which had a maximal response too close to the start or the end of the corridor $(0-15 \mathrm{~cm}$ or $85-100 \mathrm{~cm})$ were not considered for analysis of the ratio of responses. This excluded cells which responded too close to the start or the end of the corridor, which were outside the visually-matching segments Two-dimensional response profiles with respect to position and speed (Supplementary Figure 5c) were calculated as previously described (Saleem et al., 2013).

\section{Decoding population activity $\&$ using position decoded from other cells}

Population activity was decoded using an independent Bayes decoder. For every time bin, we calculated the probability of being at a location $x$ given population response $R$ as:

$$
P(x \mid R)=\frac{1}{Z} P(x)\left(\prod_{i=1}^{M} f_{i}(x)^{r_{i}}\right) \exp \left(-t \sum_{i=1}^{M} f_{i}(x)\right)
$$

where $f_{i}(x)$ is the response profile and $r_{i}$ is the spike count of the $i^{\text {th }}$ neuron in a time bin, $M$ is the number of neurons and $t$ is the time window. $Z$ is a normalizing constant, which makes the probabilities across all positions sum to one (Zhang et al., 1998; Bendor and Wilson, 2012). The probability of being in the reward zone was calculated by summing the posterior probabilities in the reward zone, and normalized relative to the value in correct trials (Figure $3 \mathrm{c}$ and $3 \mathrm{f}$ ). When calculating joint distributions (Figures 2 and 3), we smoothed the distribution by a Gaussian window with a width of 4 spatial bins. To account for the effects of position and speed on calculating the correlations between V1 and CA1 decoding errors, we shuffled the data within the time points when the animal was at the same position (within $2 \mathrm{~cm}$ ) and ran in a specific speed range $(5 \mathrm{~cm} / \mathrm{s}$ bins: $5-10 \mathrm{~cm} / \mathrm{s}$ to $30-35 \mathrm{~cm} / \mathrm{s})$. 


\section{Simulation of $\mathbf{V} 1$ complex cells}

Response profiles expected from purely visual neurons were obtained from simulations of a population of complex receptive fields. Complex receptive fields were modelled as two Gabor filters in spatial quadrature (i.e. shifted in spatial phase by $90 \mathrm{deg}$ ) having the same orientation and spatial frequency. Responses were simulated by convolving the VR images at successive positions along the corridor with the pair of Gabor filters and taking the sum of their squared outputs (energy model (Movshon et al., 1978; Carandini, 2006)). The receptive fields were designed so to simulate different orientation selectivity (from $0^{\circ}, 15^{\circ}, \ldots, 165^{\circ}$; we overrepresented the cardinal orientations) spatial frequency selectivity $(0.04,0.05,0.06$ and 0.07 cycles/deg), which are the ranges typically observed in the mouse visual system (Niell and Stryker, 2010; Andermann et al., 2011; Marshel et al., 2011). The receptive fields were simulated to cover azimuths from $40^{\circ}$ to $80^{\circ}$, matching the receptive field position of the cells we focused on in our recordings.

\section{Methods References}

Andermann ML, Kerlin AM, Roumis DK, Glickfeld LL, Reid RC (2011) Functional specialization of mouse higher visual cortical areas. Neuron 72:1025-1039.

Ayaz A, Saleem AB, Schölvinck ML, Carandini M (2013) Locomotion controls spatial integration in mouse visual cortex. Curr Biol 23:890-894.

Bendor D, Wilson MA (2012) Biasing the content of hippocampal replay during sleep. Nat Neurosci 15:14391444.

Carandini M (2006) What simple and complex cells compute. J Physiol 577:463-466 Available at: http://doi.wiley.com/10.1113/jphysiol.2006.118976.

Carandini M, Shimaoka D, Rossi LF, Sato TK, Benucci A, Knopfel T (2015) Imaging the Awake Visual Cortex with a Genetically Encoded Voltage Indicator. J Neurosci 35:53-63.

Dipoppa M, Ranson A, Krumin M, Pachitariu M, Carandini M, Harris KD (2016) Vision and locomotion shape the interactions between neuron types in mouse visual cortex. Available at:

http://biorxiv.org/lookup/doi/10.1101/058396.

Harris K, Csicsvari J, Hirase H, Dragoi G, Buzsáki G (2003) Organization of cell assemblies in the hippocampus. Nature 424:552-556 Available at:

http://www.nature.com/nature/journal/v424/n6948/abs/nature01834.html.

Harris KD, Henze D a, Hirase H, Leinekugel X, Dragoi G, Czurkó A, Buzsáki G (2002) Spike train dynamics predicts theta- related phase precession in hippocampal pyramidal cells. Nature 417:2116-2118 Available at: http://www.ncbi.nlm.nih.gov/pubmed/12066184.

Kadir SN, Goodman DFM, Harris KD (2014) High-Dimensional Cluster Analysis with the Masked EM Algorithm. Neural Comput 26:2379-2394 Available at: http://www.mitpressjournals.org/doi/10.1162/NECO_a_00661.

Kalatsky VA, Stryker MP (2003) New paradigm for optical imaging: Temporally encoded maps of intrinsic signal. Neuron 38:529-545.

Loader CR (1996) Local likelihood density estimation. Ann Stat 24:1602-1618.

Madisen $L$ et al. (2015) Transgenic mice for intersectional targeting of neural sensors and effectors with high specificity and performance. Neuron 85:942-958.

Marshel JH, Garrett ME, Nauhaus I, Callaway EM (2011) Functional specialization of seven mouse visual cortical areas. Neuron 72:1040-1054.

Movshon JA, Thompson ID, Tolhurst DJ (1978) Spatial summation in the receptive fields of simple cells in the cat's striate cortex. J Physiol 283:53-77 Available at: http://jp.physoc.org/content/283/1/53.short.

Niell CM, Stryker MP (2010) Modulation of Visual Responses by Behavioral State in Mouse Visual Cortex. Neuron 65:472-479.

Pachitariu M, Stringer C, Schröder S, Dipoppa M, Rossi LF, Carandini M, Harris KD (2016) Suite2p: beyond 10,000 neurons with standard two-photon microscopy. bioRxiv:61507 Available at:

http://biorxiv.org/lookup/doi/10.1101/061507. 
Peron SP, Freeman J, Iyer V, Guo C, Svoboda K (2015) A Cellular Resolution Map of Barrel Cortex Activity during Tactile Behavior. Neuron 86:783-799.

Pologruto TA, Sabatini BL, Svoboda K (2003) ScanImage: Flexible software for operating laser scanning microscopes. Biomed Eng Online 2:13 Available at: http://biomedical-engineeringonline.biomedcentral.com/articles/10.1186/1475-925X-2-13.

Ratzlaff EH, Grinvald A (1991) A tandem-lens epifluorescence macroscope: Hundred-fold brightness advantage for wide-field imaging. J Neurosci Methods 36:127-137.

Rossant C, Kadir SN, Goodman DFM, Schulman J, Hunter MLD, Saleem AB, Grosmark A, Belluscio M, Denfield GH, Ecker AS, Tolias AS, Solomon S, Buzsáki G, Carandini M, Harris KD (2016) Spike sorting for large, dense electrode arrays. Nat Neurosci 19:634-641 Available at: http://www.nature.com/doifinder/10.1038/nn.4268.

Saleem AB, Ayaz A, Jeffery KJ, Harris KD, Carandini M (2013) Integration of visual motion and locomotion in mouse visual cortex. Nat Neurosci 16:1864-1869 Available at: http://www.nature.com/doifinder/10.1038/nn.3567.

Steinmetz NA et al. (2017) Aberrant Cortical Activity in Multiple GCaMP6-Expressing Transgenic Mouse Lines. eneuro 4:ENEURO.0207-17.2017 Available at: http://eneuro.sfn.org/lookup/doi/10.1523/ENEURO.0207-17.2017.

Wekselblatt JB, Flister ED, Piscopo DM, Niell CM (2016) Large-scale imaging of cortical dynamics during sensory perception and behavior. J Neurophysiol 115:2852-2866 Available at: http://jn.physiology.org/lookup/doi/10.1152/jn.01056.2015.

Yang Z, Heeger DJ, Seidemann E (2007) Rapid and Precise Retinotopic Mapping of the Visual Cortex Obtained by Voltage-Sensitive Dye Imaging in the Behaving Monkey. J Neurophysiol 98:1002-1014 Available at: http://jn.physiology.org/cgi/doi/10.1152/jn.00417.2007.

Zhang K, Ginzburg I, McNaughton BL, Sejnowski TJ (1998) Interpreting neuronal population activity by reconstruction: unified framework with application to hippocampal place cells. J Neurophysiol 79:1017-1044 Available at: http://jn. physiology.org/content/79/2/1017.abstract. 